\title{
Gut Microbiota in Inflammatory Bowel Disease
}

\author{
Jung Ok Shim \\ Department of Pediatrics, Korea University College of Medicine, Seoul, Korea
}

The gut mucosal barrier plays an important role in maintaining a delicate immune homeostasis. The pathogenesis of inflammatory bowel disease (IBD) is considered to involve a defective mucosal immunity along with a genetic predisposition. Recent views have suggested an excessive response to components of the gut microbiota in IBD. A condition of "dysbiosis", with alterations of the gut microbial composition, has been observed in patients with IBD. In this article, the author review recent studies of gut microbiota in IBD, particularly the importance of the gut microbiota in the pathogenesis of pediatric IBD. (Pediatr Gastroenterol Hepatol Nutr 2013; 16: $17 \sim 21$ )

Key Words: Microbiota, Inflammatory bowel diseases, Child

\section{INTRODUCTION}

Different genetic, immunologic, and environmental factors are implicated in the pathogenesis of inflammatory bowel disease (IBD). Recent views have suggested that patients with IBD may exhibit an excessive response to components of the gut microbiota. The gut mucosal barrier plays an important role in maintaining a delicate immune homeostasis. An inadequate immune response or overreaction of the immune system against food antigens ( $\mathrm{Ag})$ or components of the commensal flora can be a threat to this homeostasis.

The human gut contains as many as $10^{14}$ individual bacteria, comprising more than 1,000 different species. The most abundant bacteria phyla found in the large intestine of healthy adults are gram-negative Bacteroidetes and gram positive Firmicutes [1]. The intestinal microbiota are considered to act as a "metabolic organ", by deriving energy from nutrients that escape digestion in the upper gut. Moreover, the microbiota are considered to exert a barrier effect against pathogens in the intestinal mucosa. Germ-free animals do not develop enterocolitis when raised under germ-free conditions. However, when the animals are exposed to microbiota, mild to severe enterocolitis develops [2]. These results indicate the importance of gut microbiota in the pathogenesis of IBD.

\section{DYSBIOSIS AND ABNORMAL IMMUNE RESPONSE}

The concept of "dysbiosis" was introduced many

Received : March 14, 2013, Revised : March 18, 2013, Accepted : March 20, 2013

Corresponding author: Jung Ok Shim, Department of Pediatrics, Korea University Guro Hospital, 148, Gurodong-ro, Guro-gu, Seoul 152-703, Korea. Tel: +82-2-2626-3157, Fax: +82-2-2626-1249, E-mail: shimjo@korea.ac.kr

Copyright (c) 2013 by The Korean Society of Pediatric Gastroenterology, Hepatology and Nutrition

This is an open-access article distributed under the terms of the Creative Commons Attribution Non-Commercial License (http://creativecommons.org/licenses/by-nc/3.0/) which permits unrestricted non-commercial use, distribution, and reproduction in any medium, provided the original work is properly cited. 
decades ago, and was initially defined as an increase of enteric pathogen and decrease of commensal bacteria; however neither its biologic basis nor the concept of normal microbial balance has been defined. Technical development from a culture-dependent era to metagenomics has led to advances in the study of intestinal microbiota. The healthy host has an immune defense mechanism and tolerance toward microbe, and maintains immune homeostasis. Dysregulation of host-microbe interaction can lead to inadequate or hyperinflammatory reactions.

Intestinal epithelial cells provide a physical barrier between the luminal microbes and the intestinal tissues. Epithelial cells produce a mucus layer and secrete antimicrobial proteins that limit bacterial exposure to the epithelial cells. Secretory immunoglobulin A (IgA) gives protection from microbes. Microbial sensing is mediated through Toll-like receptors (TLRs) and nucleotide-binding domain and leucine-rich repeat-containing receptors (NLRs). The activation of TLRs and NLRs mediates microbial killing. Defense mechanisms that protect intestinal tissues from microbes also act as tolerance mechanisms. Activation of TLRs and NLRs on macrophages and dendritic cells (DCs) in the intestinal lamina propria does not produce proinflammatory cytokines. DCs present the antigen to $\mathrm{T}$ cells in the Peyer's patches, which can activate differentiation of regulatory $\mathrm{T}$ (Treg) cells, regulated by interleukin-10 (IL-10), transforming growth factor-beta (TGF- $\beta$ ), and retinoic acid. Thymic stromal lymphopoietin and other factors produced by epithelial cells can lead to immune tolerance [3].

Overgrowth of aggressive commensal microbes increases the number of antigens $(\mathrm{Ag})$ that induce pathogenic immune responses or increase mucosal permeability. Increased mucosal permeability cause pathogenic innate and T-cell immune responses. Decreased production of secretory IgA can lead to bacterial overgrowth. Defective killing of phagocytosed bacteria can lead to ineffective clearance of bacterial Ag. Antigen-presenting cells and epithelial cells overproduce cytokines due to ineffective down-regulation, which results in $\mathrm{T}$ helper cell (Th) 1 and Th17 differentiation and inflammation. Defective Treg cells cause decreased secretion of IL-10 and TGF- $\beta$, and loss of immune tolerance to microbial Ag [4].

\section{GUT MICROBIOTA AND IBD}

Both quantitative and qualitative changes in the microbial composition have been reported in IBD. Previous studies have shown decreased diversity and an increased intestinal mucosal-adhesive microbes [5-7]. Manichanh et al. [8] performed 16S ribosomal ribonucleic acid (rRNA) metagenome analysis of microbiota in feces of patients with Crohn's disease. They noted that the most abundant bacteria were Firmicutes and Bacteroidetes; however, the number of ribotypes of Firmicutes was decreased to 13 , compared with 43 ribotypes in healthy adult-control individuals. In particular, diversity in Clostridium leptum was decreased predominantly. Seksik et al. [9] examined intestinal microbiota in IBD according to the disease activity. They reported decreased diversity of Bacteroides in active state Crohn's disease compared with those in remission state disease and in healthy individuals. A pyrosequencing study in twins has shown that intestinal microbial profiles vary with IBD phenotypes [10]. They reported increased diversity of Firmicutes in patients with colonic Crohn's disease, and decreased diversity in patients with ileal Crohn's disease. Decreased diversity of bifidobacteria and lactobacilli in the feces of patients with IBD have been reported in some studies [11,12].

The intestinal microbiota present in the feces and in the mucosal or intraepithelial tissue are different. Microbiota that directly interact with the intestinal mucosa can be obscured by fecal flora. A greater abundance in Enterobacteriaceae, mostly Escherichia coli, was observed in patients with Crohn's disease, more notably in mucosa-associated microbiota as compared to fecal sam- 
ples [13]. In addition, decreased diversity in Feacalibacteria (Faecalibacterium prausnitzii) in mucosa-assosiated microbiota or fecal samples has been reported. Microbial analyses of specimens indicate that the ratio of $F$. prausnitzii/E. coli could be used to evaluate the level of the dysbiosis in IBD [14]. Negroni et al. [15] reported 2 new strains of adhesive-invasive $E$. coli (AIEC) in the tissues of children with IBD. These AIEC upregulated CEACAM6, tumor necrosis factor- $\alpha$, and IL-8 gene/protein expression, and could also survive inside macrophages and damage epithelial barrier integrity. Higher concentrations of mucosa-associated bacteria (proteobacteria and actinobacteria) in Crohn's disease and ulcerative colitis have been reported. Frank et al. [16] performed rRNA sequence analysis of surgical specimen obtained from IBD patients. The results were characterized by the depletion of Firmicutes groups $14 \mathrm{a}$ and 4 (Lachnospiraceae) and Bacteroidetes, and an increased concentration of proteobacteria. However, the increase of proteobacteria was relflective of a relative change in the composition, primarily due to decrease in the composition of Firmicutes and Bacteroidetes.

Mycobacterium avium substrain paratuberculosis (MAP), AIEC, Yersinia, and Pseudomonas are reportedly associated with the development of Crohn's disease [17]. Clostridium difficile, Entamoeba histolytica, Campylobacter, adenovirus, rotavirus, and mycoplasma were not primary causes, but could be associated with relapse. $C$. difficile was detected in $10-20 \%$ of patients with reactivated IBD [18].

In total, the $20-25 \%$ of patients with IBD develop symptoms during childhood. Genetic factors are more influential than exogenous factors in children. In particularly, infants have immature mucosal immune systems and the intestinal microbiota composition is constantly changing. Few studies are available in pediatric patients with IBD. Recently, Michail et al. [19] reported decreased diversity of microbes in the feces of 27 children with ulcerative colitis. In children, depletion of Firmicutes, Verrucomicrobiae, and Lentis- phaerae was prominent, a finding that was not different from the data of adults. It is possible that because most of the patients examined were adolescents, further study is needed in infants and young children. Michail et al. also reported that the decreased diversity of microbes was more prominent in the steroid non-responder group than in the steroid responder group.

\section{MICROBIOTA AND GENETIC PREDIS- POSITION}

Host genetics can contribute to dysbiosis. Mutations in specific genes can lead to abnormal immune regulation, and in turn, may affect microbial composition. The importance of genetic predisposition for the development of IBD is well known. Since the discovery in 2001 that polymorphisms in the NOD2/CARD15 gene predispose to Crohn's disease in Caucasians [20], genome-wide association studies have identified at least 70 susceptibility loci for Crohn's disease and 40 loci for ulcerative colitis. NOD2 is an intracellular pathogen-recognition receptor for bacterial muramyl dipeptide within mononuclear phagocytes and intestinal epithelial cells. ATG16L1 and IRGM genes act as susceptibility factors that mediate autophagy, which is a degradation system that delivers intracellular debris to lysosomes and contributes to the elimination of intracellular bacteria. A number of the candidate genes are of interest with regard to their influence on acute inflammatory response, including those associated with mucosal barrier function, cytokines or their related proteins (IL-10, IL-19, IL-27, IL-1RL1, IL-2RA, IL-12RL2, and IL-18R1), and chemokines (CCL 2 and CCL7). Almost all candidate genes participate in the host immune and adaptive response to microbial organisms and in the maintenance of homeostasis [21].

NOD2 variants (L1007X, R702W, G908R) and a ATG16L1 variant (T300A) were reportedly associated with dysbiosis in resected tissue from IBD patients, with decreases in Clostridium and in- 
creases in other bacteria in Crohn's disease and ulcerative colitis [22]. No associations were observed between NOD2 variants, the presence of MAP, and incidence of IBD [23].

\section{CONCLUSION}

Dysbiosis is an important immunologic pathogenenic process in IBD. Changes of intestinal microbiota through genetics and environment (such as diet, infections or antibiotics) may contribute to defective host immune response. Dysbiosis and immune dysregulation might have a greater influence in young children than adolescents or adults. However, it is not clear whether dysbiosis contributes to the development of IBD or is instead a consequence of the disease.

Despite the importance of microbiota, antibiotics were not effective in the treatment of IBD, except in specific circumstances. Fecal transplantation has been attempted for fulminant and refractory $C$. difficile and in a few patients with IBD [24]. Further study is needed to evaluate the diagnostic and therapeutic implications in IBD.

\section{REFERENCES}

1. Seo JK. Pediatric inflammatory bowel disease (IBD): Phenotypic, genetic and therapeutic differences between early-onset and adult-onset IBD. Korean J Pediatr Gastroenterol Nutr 2011;14:1-25.

2. Taurog JD, Richardson JA, Croft JT, Simmons WA, Zhou M, Fernández-Sueiro JL, et al. The germfree state prevents development of gut and joint inflammatory disease in HLA-B27 transgenic rats. J Exp Med 1994;180:2359-64.

3. Abraham C, Medzhitov R. Interactions between the host innate immune system and microbes in inflammatory bowel disease. Gastroenterology 2011;140: 1729-37.

4. Aldhous MC. Gene-environmental interactions in inflammatory bowel disease: microbiota and genes. Frontline Gastroenterology 2012;3:180-6.

5. Thomazini CM, Samegima DA, Rodrigues MA, Victoria CR, Rodrigues J. High prevalence of aggregative adherent Escherichia coli strains in the mucosa-associated microbiota of patients with inflammatory bowel diseases. Int J Med Microbiol 2011;301:475-9.

6. Lapaquette P, Glasser AL, Huett A, Xavier RJ, Darfeuille-Michaud A. Crohn's disease-associated adherent-invasive E. coli are selectively favoured by impaired autophagy to replicate intracellularly. Cell Microbiol 2010;12:99-113.

7. Andersson U, Wang H, Palmblad K, Aveberger AC, Bloom O, Erlandsson-Harris H, et al. High mobility group 1 protein (HMG-1) stimulates proinflammatory cytokine synthesis in human monocytes. J Exp Med 2000;192:565-70.

8. Manichanh C, Rigottier-Gois L, Bonnaud E, Gloux K, Pelletier E, Frangeul L, et al. Reduced diversity of faecal microbiota in Crohn's disease revealed by a metagenomic approach. Gut 2006;55:205-11.

9. Seksik P, Rigottier-Gois L, Gramet G, Sutren M, Pochart P, Marteau P, et al. Alterations of the dominant faecal bacterial groups in patients with Crohn's disease of the colon. Gut 2003;52:237-42.

10. Willing BP, Dicksved J, Halfvarson J, Andersson AF, Lucio M, Zheng Z, et al. A pyrosequencing study in twins shows that gastrointestinal microbial profiles vary with inflammatory bowel disease phenotypes. Gastroenterology 2010;139:1844-54.e1.

11. Hammer HF. Gut microbiota and inflammatory bowel disease. Dig Dis 2011;29:550-3.

12. Iebba V, Aloi M, Civitelli F, Cucchiara S. Gut microbiota and pediatric disease. Dig Dis 2011;29:531-9.

13. Swidsinski A, Ladhoff A, Pernthaler A, Swidsinski S, Loening-Baucke V, Ortner M, et al. Mucosal flora in inflammatory bowel disease. Gastroenterology 2002;122: 44-54.

14. Chassaing B, Darfeuille-Michaud A. The commensal microbiota and enteropathogens in the pathogenesis of inflammatory bowel diseases. Gastroenterology 2011; 140:1720-8.

15. Negroni A, Costanzo M, Vitali R, Superti F, Bertuccini L, Tinari A, et al. Characterization of adherent-invasive Escherichia coli isolated from pediatric patients with inflammatory bowel disease. Inflamm Bowel Dis 2012; 18:913-24.

16. Frank DN, St Amand AL, Feldman RA, Boedeker EC, Harpaz N, Pace NR. Molecular-phylogenetic characterization of microbial community imbalances in human inflammatory bowel diseases. Proc Natl Acad Sci U S A 2007;104:13780-5.

17. Nagalingam NA, Lynch SV. Role of the microbiota in inflammatory bowel diseases. Inflamm Bowel Dis 2012; 18:968-84.

18. Meyer AM, Ramzan NN, Loftus EV Jr, Heigh RI, 
Leighton JA. The diagnostic yield of stool pathogen studies during relapses of inflammatory bowel disease. J Clin Gastroenterol 2004;38:772-5.

19. Michail S, Durbin M, Turner D, Griffiths AM, Mack DR, Hyams J, et al. Alterations in the gut microbiome of children with severe ulcerative colitis. Inflamm Bowel Dis 2012;18:1799-808.

20. Hugot JP, Chamaillard M, Zouali H, Lesage S, Cézard JP, Belaiche J, et al. Association of NOD2 leucine-rich repeat variants with susceptibility to Crohn's disease. Nature 2001;411:599-603.

21. Maloy KJ, Powrie F. Intestinal homeostasis and its breakdown in inflammatory bowel disease. Nature 2011;474:298-306.
22. Frank DN, Robertson CE, Hamm CM, Kpadeh Z, Zhang $\mathrm{T}$, Chen $\mathrm{H}$, et al. Disease phenotype and genotype are associated with shifts in intestinal-associated microbiota in inflammatory bowel diseases. Inflamm Bowel Dis $2011 ; 17: 179-84$.

23. Brant SR, Wang MH, Rawsthorne P, Sargent M, Datta LW, Nouvet F, et al. A population-based case-control study of CARD15 and other risk factors in Crohn's disease and ulcerative colitis. Am J Gastroenterol 2007;102:313-23.

24. Landy J, Al-Hassi HO, McLaughlin SD, Walker AW, Ciclitira PJ, Nicholls RJ, et al. Review article: faecal transplantation therapy for gastrointestinal disease. Aliment Pharmacol Ther 2011;34:409-15. 